\title{
THD Doppler procedure for hemorrhoids: the surgical technique
}

\author{
C. Ratto
}

Received: 10 July 2013/Accepted: 6 August 2013/Published online: 12 September 2013

(C) Springer-Verlag Italia 2013

\begin{abstract}
Transanal hemorrhoidal dearterialization (THD) is an effective treatment for hemorrhoidal disease. The ligation of hemorrhoidal arteries (called "dearterialization") can provide a significant reduction of the arterial overflow to the hemorrhoidal piles. Plication of the redundant rectal mucosa/submucosa (called "mucopexy") can provide a repositioning of prolapsing tissue to the anatomical site. In this paper, the surgical technique and perioperative patient management are illustrated. Following adequate clinical assessment, patients undergo THD under general or spinal anesthesia, in either the lithotomy or the prone position. In all patients, distal Doppler-guided dearterialization is performed, providing the selective ligation of hemorrhoidal arteries identified by Doppler. In patients with hemorrhoidal/muco-hemorrhoidal prolapse, the mucopexy is performed with a continuous suture including the redundant and prolapsing mucosa and submucosa. The description of the surgical procedure is complemented by an accompanying video (see supplementary material). In long-term follow-up, there is resolution of symptoms in the vast majority of patients. The most common complication is transient tenesmus, which sometimes can result in rectal discomfort or pain. Rectal bleeding occurs in a very limited number of patients. Neither fecal incontinence nor chronic pain should occur.
\end{abstract}

Electronic supplementary material The online version of this article (doi:10.1007/s10151-013-1062-3) contains supplementary material, which is available to authorized users.

C. Ratto $(\bowtie)$

Department of Surgical Sciences, Catholic University, Largo A. Gemelli, 8, 00168 Rome, Italy

e-mail: carloratto@tiscali.it
Anorectal physiology parameters should be unaltered, and anal sphincters should not be injured by following this procedure. When accurately performed and for the correct indications, THD is a safe procedure and one of the most effective treatments for hemorrhoidal disease.

Keywords Hemorrhoids · Dearterialization · Mucopexy · Hemorrhoidal artery · Prolapse · Recurrence $\cdot$ Complication

\section{Introduction}

Recent findings concerning the pathophysiology of the hemorrhoidal disease [1-4], and the development of new technologies for surgical treatment [5], have favored a rapid spread of an innovative approach, the ligation of hemorrhoidal arteries, with or without pexy of prolapsing rectal mucosa/submucosa. A number of procedures have been devised using Doppler guidance and different surgical devices. Recent reviews [6, 7] have evaluated these techniques grouping them together, generating some confusion between different procedures. This paper provides an overview of the technical aspects and perioperative management of one of the most widely used techniques, transanal hemorrhoidal dearterialization (THD). This surgical procedure is primarily oriented toward the management of the main symptoms of hemorrhoidal disease (i.e., bleeding, prolapse, and pain), intervening on its pathophysiological processes. THD is based on two technical steps: (1) the targeted ligation of hemorrhoidal arteries (called "dearterialization"), using a very sensitive continuous Doppler probe able to identify the maximal flow; (2) the plication and lifting of redundant and prolapsing rectal mucosa/submucosa (called "mucopexy"). 


\section{Patient assessment}

An accurate assessment of patient's history is mandatory, particularly concerning symptoms related to hemorrhoidal disease. Then, both anorectal examination and anoscopy are carried out to evaluate hemorrhoidal engorgement, spontaneous bleeding, and eventual prolapse of piles and rectal mucosa/submucosa, both at rest and during straining. In particular, reducibility of hemorrhoidal prolapse should be assessed. Anal skin tags should also be noted and distinguished from real hemorrhoidal prolapse. Other anal and/or rectal diseases and functional disorders must be diagnosed/excluded. In particular, patients complaining of symptoms of obstructed defecation should be further investigated. Finally, endoscopic assessment of the colon and rectum should be performed according to the guidelines for colorectal cancer screening.

\section{Indications}

Transanal hemorrhoidal dearterialization should be reserved for patients presenting active hemorrhoidal disease despite lifestyle/diet interventions, drug therapy, and minor office procedures such as rubber band ligation or sclerotherapy. Indications should be established on the basis of the patient's symptoms and physical findings. If the main complaint is bleeding, this can be addressed by dearterialization alone, ligating of the hemorrhoidal arteries along the low rectal circumference. Usually, at least 6 arteries are found and ligated using the THD Doppler device. In case of bleeding associated with hemorrhoidal or mucosal and hemorrhoidal prolapse, mucopexy should be added to the dearterialization. In fact, mucopexy can be regarded as an "on-demand" step of THD, depending also on the location and severity of mucosal prolapse (in terms of its length). It is mandatory that the prolapsing hemorrhoidal piles and rectal mucosa should be reducible, so that they will reach their respective anatomical sites. Therefore, fibrosed piles cannot be treated with THD. When the prolapse involves the whole rectal circumference, 6 separate mucopexy sutures may be placed. Alternatively, if there is only limited circumferential involvement, a smaller number of running sutures should be used. Patients, who complain of mucosal and hemorrhoidal prolapse or hemorrhoidal prolapse alone, usually have a history of bleeding, which disappeared in the later phase of hemorrhoidal disease in accordance with the pathophysiological evolution of the disease. These patients should undergo both dearterialization and mucopexy following the same criteria mentioned above. Mucopexy can be adapted to different lengths of mucosal prolapse, making longer or shorter running sutures. However, attention must be paid to misdiagnosed internal rectal intussusception, which is not amenable to mucopexy used for the hemorrhoidal prolapse. According to the Goligher's classification, 1st degree or initial 2nd degree hemorrhoids, unresponsive to conservative treatment or minimal surgery, may be addressed by dearterialization alone. More advanced 2nd degree, 3rd degree, and 4th degree (except in the case of fixed, fibrotic piles) should undergo dearterialization and mucopexy.

Patients with skin tags should be advised that these are not real hemorrhoids, but the consequence of previous engorgement and dislodgement of hemorrhoidal cushions toward the perianal skin. Since THD does not provide any specific treatment for skin tags, only surgical excision can be a reliable treatment when indicated or desired.

Patients with hemorrhoids who suffer from inflammatory bowel disease deserve a special mention. There is a lack of studies specifically addressing patients with Crohn's disease or ulcerative colitis operated on with THD. However, providing that no severely active inflammation is demonstrated on the rectal mucosa, this method may be suitable in patients with hemorrhoids resistant to conservative treatments. The same concept applies to hemorrhoidal disease in patients with chronic radiation proctitis.

\section{Preparation for surgery}

This is a matter of the surgeon's preference as there are no absolute guidelines in hemorrhoidal surgery. The same is true also for the THD procedure. Because it is performed within the lower rectum, one or two enema(s) should be prescribed. The Author does not consider antibiotic prophylaxis as mandatory as in his experience no infections have been observed following this operation.

\section{Anesthesia}

Transanal hemorrhoidal dearterialization can be performed under both general and locoregional anesthesia. Propofolremifentanil anesthesia, with the placement of a laryngeal mask, combines general anesthesia, complete control of vital parameters, and quick reversion and discharge from the hospital. Spinal anesthesia may be limited to the most caudal metameric nerve roots avoiding any prolonged stay in bed. Unfortunately, spinal anesthesia is usually associated with a higher risk of urinary retention, especially following hemorrhoid surgery. More limited locoregional anesthesia (i.e., posterior perianal block) does not ensure a complete intraoperative analgesia due to the visceral pain elicited by surgical ligation, suturing for plication, and tying knots on the rectal mucosa. 


\section{Intraoperative management}

The patient can be placed in either the lithotomy or the prone position, based on the surgeon's preference. However, it should be taken into consideration that the lithotomy position allows a more realistic position of the prolapsing hemorrhoids and rectal mucosa. An accurate intraoperative monitoring of blood pressure could be helpful. In particular, systolic pressure higher than $100-110 \mathrm{mmHg}$ allows auscultation of a Doppler signal necessary for the identification of the hemorrhoidal arteries.

\section{Equipment}

Transanal hemorrhoidal dearterialization is performed using a specific device produced by THD S.p.A., Correggio, Italy. It consists of a proctoscope equipped with a Doppler probe and a light source (Fig. 1). The Doppler probe utilizes a double crystal, which allows a more precise focusing of the ultrasound waves and capturing of largediameter arteries located in the superficial layers of the rectal wall. Sufficient space is provided around two crystals for their adequate vibration. The Doppler probe is mounted on an oblique support, oriented toward the operative window, so that the artery identified by the Doppler signal lies within the operative window and can be selectively ligated.

The latest proctoscope model (THD Slide ${ }^{\circledR}$, THD S.p.A., Correggio, Italy) has a sliding part comprising the operating window and the Doppler probe, so that the operator can move them proximally and distally without repositioning the proctoscope. The section of the proctoscope is elliptical, with an external maximum diameter of $32-34 \mathrm{~mm}$ and an internal diameter of 20-34 mm.

The recommended suture is $2-0$ absorbable polyglycolic acid with a $5 / 8$-in. needle. This is mounted on a specially designed needle holder, providing a mark on the tip where the needle should be held. With this configuration, the needle holder tip can be inserted into the pivot, and the

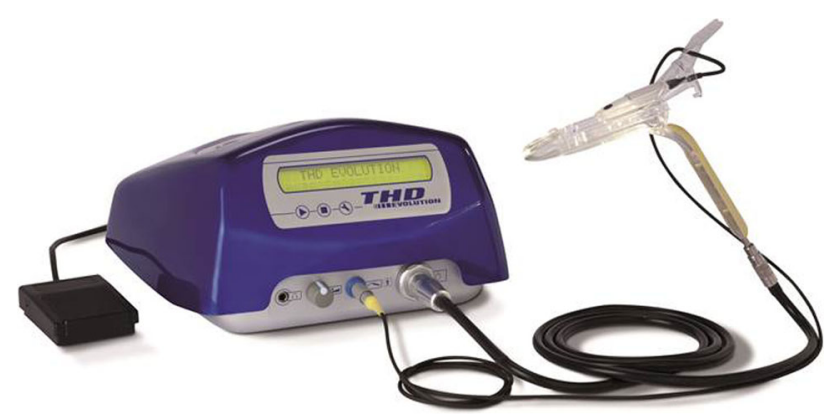

Fig. 1 Surgical instruments specifically designed for the THD procedure needle rotates to transfix the rectal mucosa in a standard fashion. The depth of the transfixed stitches can be easily and safely calibrated up to a maximum depth of $6.5 \mathrm{~mm}$, which includes only mucosa and submucosa avoiding penetration through the full thickness of the rectal wall and therefore lowering the risk of perirectal fistula and abscess. A knot-pusher is also provided in case is needed.

\section{Distal Doppler-guided dearterialization (DDD)}

Surgical anatomy of the hemorrhoidal arteries

Aim of hemorrhoidal dearterialization is to significantly reduce the arterial overflow in the hemorrhoidal tissue, characteristic of patients with hemorrhoidal disease. The anatomical and physiological characteristics of hemorrhoids have not been fully elucidated. Microscopically, hemorrhoidal tissue is composed of sinusoids, i.e., vascular structures without a muscular wall [8]. Direct arteriovenous communications have been demonstrated histologically and radiologically, and some authors have noted a resemblance to erectile tissue [9]. Traditionally, with the patient in the lithotomy position, hemorrhoids frequently appear to be localized to the left lateral, right posterolateral, and right anterolateral areas of the anal canal. However, this configuration is demonstrated in less than $20 \%$ of patients [10]. In reality, a wider network of arterial and venous vessels has been described [11]. Schuurman et al. [1] studied 10 non-fixed human cadavers in order to assess the arterial vasculature of the rectum and arterial supply to the hemorrhoids. Selective injections of different colors were used. The authors found that, about $2-3 \mathrm{~cm}$ above the dentate line, thin tortuous arteries (a mean number of 8 , all branches of the superior hemorrhoidal artery) were seen lying in the submucosa, reaching into the hemorrhoidal tissue. Smaller branches from these arteries formed a plexus in the corpus cavernosum recti area.

In our recent study [12], the majority of arteries in the upper part of the lower third of the rectum $(4-6 \mathrm{~cm}$ from the anorectal junction) were located outside the rectal wall. In contrast, within $2 \mathrm{~cm}$ from the anorectal junction, hemorrhoidal arteries were detected in the submucosa in $98 \%$ of the 6 sectors of the rectal circumference (96.6 and $100 \%$ of sectors at 2 and $1 \mathrm{~cm}$ above the anorectal junction, respectively). Therefore, in their course through the lower third of the rectum, the hemorrhoidal arteries traverse the muscularis propria of the rectum and become more superficial. These features can be easily confirmed during Doppler-guided surgical procedures. The different Doppler signals are dependent on the position of the artery (perirectal, perforating the rectal muscle, or submucosal), the distance from the Doppler probe, and the direction of 


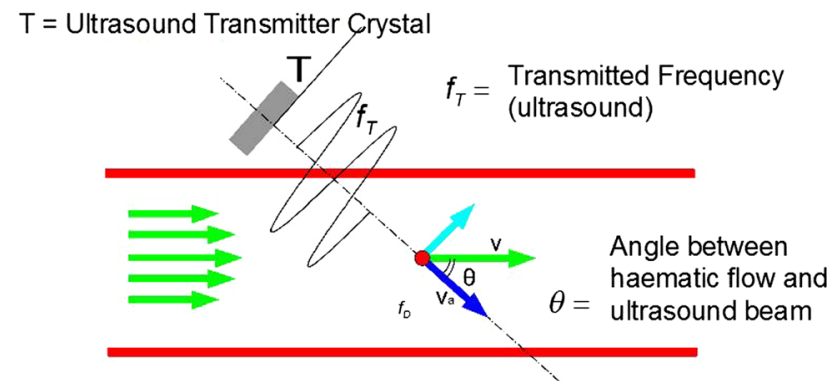

Fig. 2 Schema justifying different Doppler signals occurring during THD as related to arterial blood flow. According to this physical law, the intensity of the Doppler signal is the inverse of the cosine of the angle between the ultrasound waves and blood flow. The more perpendicular the blood flow to the ultrasound waves (i.e., artery into the perirectal tissue or submucosa) the higher the Doppler signal; the more parallel the flow (i.e., artery perforating the rectal muscle) the lower the signal

blood flow in relation to the ultrasound waves emitted by the probe. In fact, the intensity of the Doppler signal is the inverse of the cosine of the angle between the ultrasound waves and blood flow. As a consequence, the more perpendicular the blood flow to the ultrasound waves (i.e., artery into the perirectal tissue or submucosa) the higher the Doppler signal. On the other hand, the more parallel the flow (i.e., artery traversing the rectal muscle) the lower the signal (Fig. 2). The proximity of the artery to the probe when the artery is submucosal makes the Doppler signal higher than that perceived at the proximal sites.

\section{Technique}

Following gel lubrication, the proctoscope is inserted through the anal canal reaching the low rectum, about 6-7 $\mathrm{cm}$ from the anal verge. The surgeon can decide to start the operation at any point of the rectal circumference and proceed in a clockwise or anticlockwise direction. The Doppler system is then turned on. The Doppler signal corresponding to all 6 main trunks of the hemorrhoidal arteries, which are usually located at 1, 3, 5, 7, 9, and 11 o'clock of the low rectal circumference, is sought by slowly rotating and/or tilting the proctoscope. However, searching with the Doppler probe makes possible correct identification of those arteries not located at the usual odd hours positions. The proctoscope is pulled slowly back to follow the artery distally up to hemorrhoidal apex, and the best Doppler signal is searched for. According to the above-mentioned features from our previous study [12], the Doppler signal is quite clear at the proximal site (corresponding to the proximal part of the lower rectum, where, however, arteries could lie in the perirectal fat), attenuated or absent at the intermediate site (where the artery is

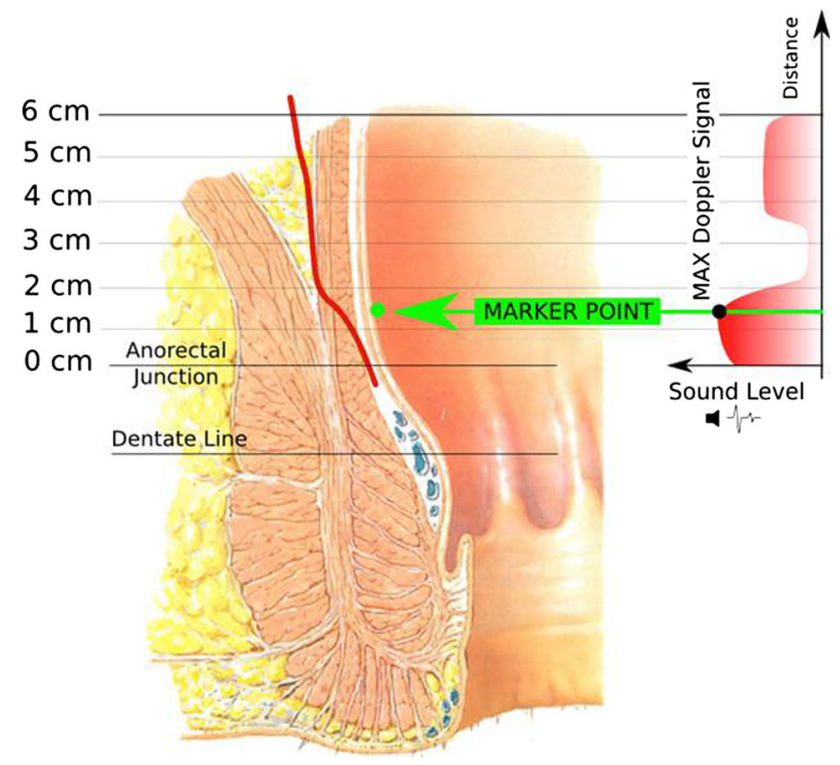

Fig. 3 Schema of the anatomical course of a hemorrhoidal artery and different levels of Doppler signal related to the position of the artery

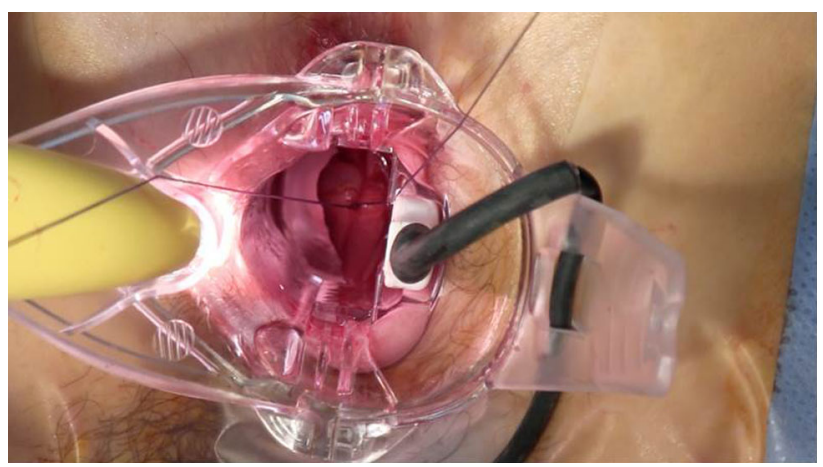

Fig. 4 Suture of a hemorrhoidal artery during DDD procedure

perforating the rectal muscle), and again clear at the distal site (within the most distal $2 \mathrm{~cm}$ of lower rectum, where the artery lies in the rectal submucosa, just above the internal hemorrhoidal piles, Fig. 3). As a consequence of anatomical and acoustic findings, the best place to find the hemorrhoidal arteries should be the most distal part of the rectum: This is the fundamental principle of distal Dopplerguided dearterialization (DDD) [13]. After identification of the best place for artery ligation, the Doppler system is turned off.

If the patient is a candidate for dearterialization alone (i.e., the patient only has bleeding without prolapse), the artery, once identified, can be directly ligated with a "Zstitch" at the site of the best Doppler signal (Fig. 4). When the patient needs to undergo dearterialization and mucopexy (due to hemorrhoidal or muco-hemorrhoidal prolapse), the rectal mucosa can be marked with electrocautery ("marker point") at the site of the best Doppler 


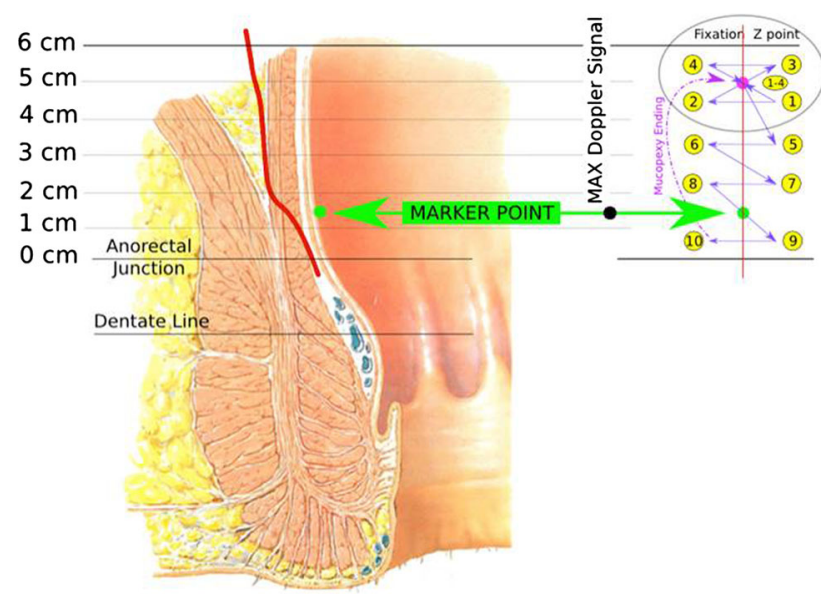

Fig. 5 Schema of mucopexy fixation point and continuous suture

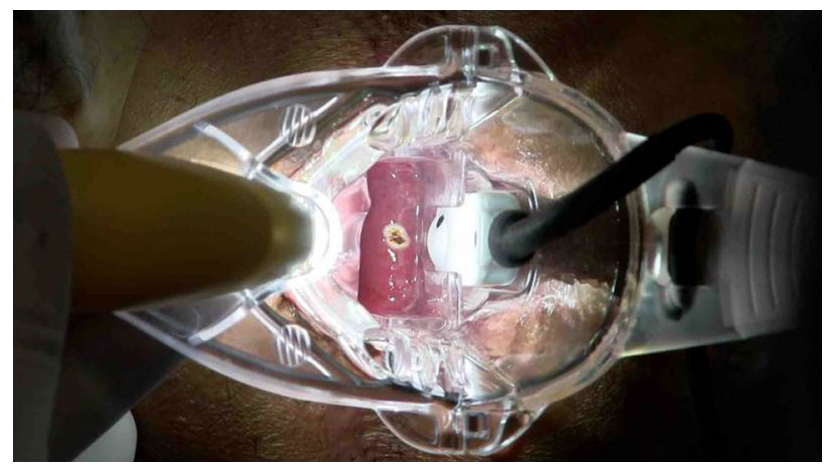

Fig. 6 "Marker point" on the distal rectal mucosa to identify the best Doppler signal obtained from the submucosal hemorrhoidal artery

signal (Figs. 5, 6) to indicate where the artery will be ligated. Then, a mucopexy follows (see below).

\section{Mucopexy (MP)}

Pathological anatomy of hemorrhoidal prolapse

Normally, the hemorrhoidal cushions are loosely attached to the circular muscle through the elastic rectal submucosa, which keeps the piles in the anal canal at rest. During defecation, rolling of the hemorrhoids inside the lumen occurs, favored by the internal anal sphincter relaxation. The fecal bolus has a shearing effect on the cushions and facilitates their prolapse [14-16]. On the other hand, the elasticity of the rectal submucosa keeps the piles inside the rectum. In patients with hemorrhoidal disease, due to altered defecation and other predisposing factors [17], the rectal submucosa progressively loses its elasticity, determining hemorrhoidal prolapse $[15,16,18]$. The progressive disruption of both the connective tissue stroma (Park's ligaments) and anchoring system (Treitz's muscle) plays a major role. Severity of prolapse is related to persistence of pathogenic factors, engorgement of piles, and progressive loss of the elasticity of the rectal submucosa.

Transanal hemorrhoidal dearterialization with mucopexy provides plication of the rectal submucosa affected by the loss of elasticity. It is reduced stably into the rectal ampulla, recovering its anatomical position. Furthermore, the scarring process induced by the mucopexy attaches the plicated mucosa and submucosa to the underlying rectal muscle.

\section{Technique}

Following the identification of the hemorrhoidal artery, the proctoscope is again pushed fully inside the distal rectum, and a "Z-stitch" is made as a proximal "fixation point" of MP. The circular device pivot can be used to do this. The proximal end of MP is not standard, depending on the length of prolapsing mucosa and submucosa. Then, the knot is tied (Fig. 7). Thereafter, the main proctoscope remains in place, and only its sliding part is moved back, exposing the rectal mucosa so that MP can be performed under direct vision. MP is carried out with a continuous

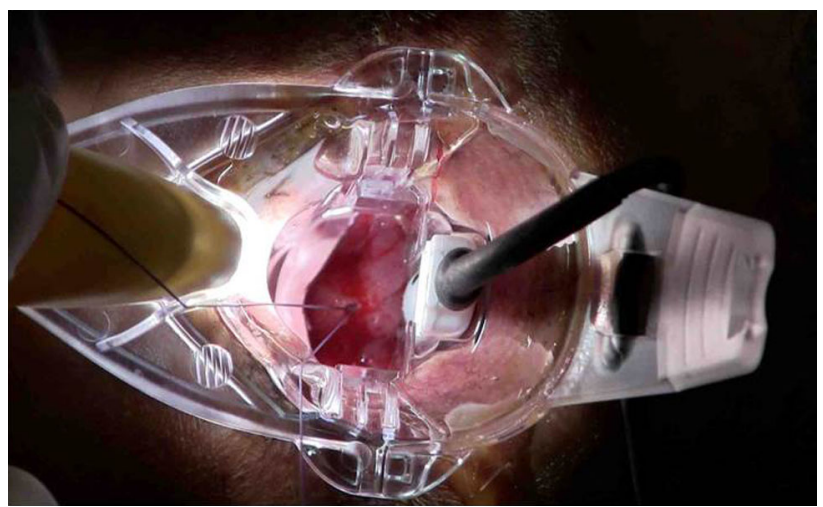

Fig. 7 Fixation $\mathrm{Z}$ point at the proximal edge of the mucopexy continuous suture

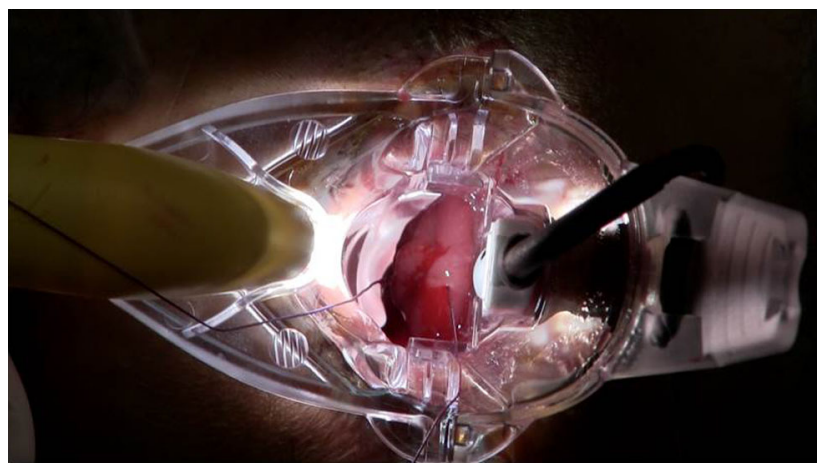

Fig. 8 Mucopexy continuous suture 
Fig. 9 Passages of the mucopexy continuous suture above (a) and below (b) the marker point to entrap the hemorrhoidal artery
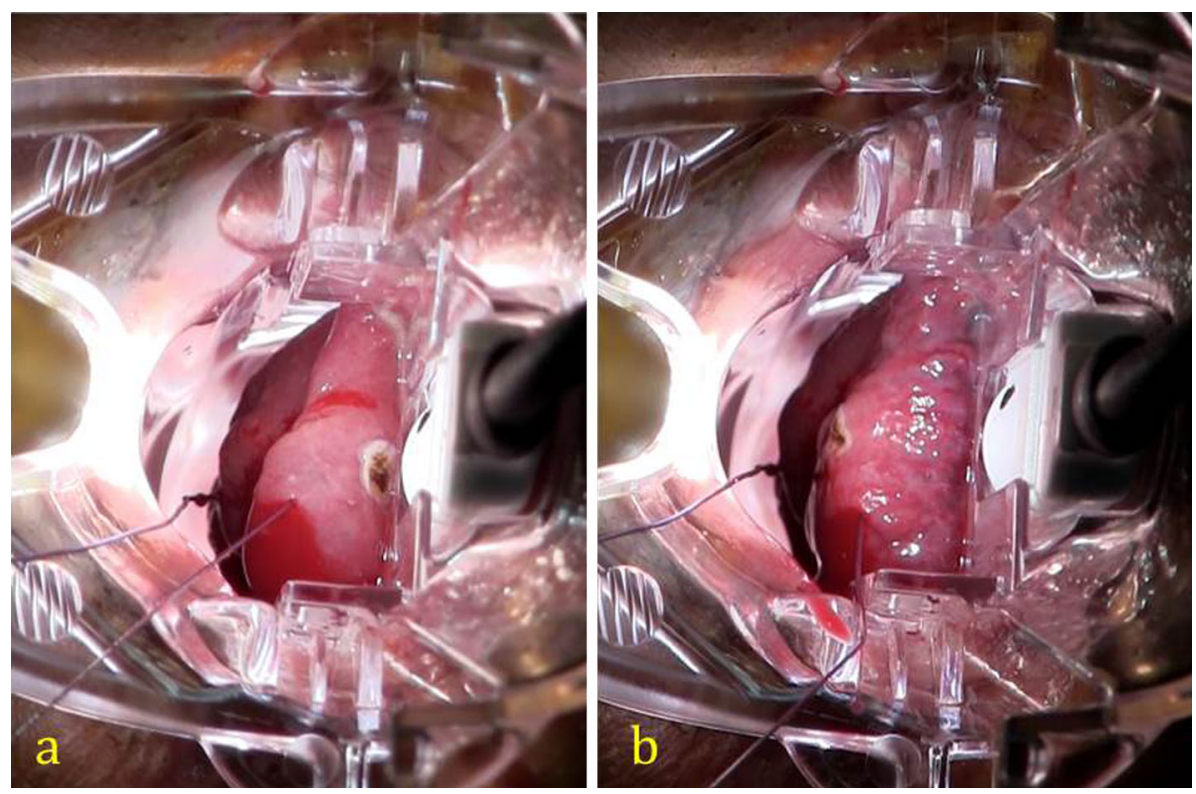

suture, including the redundant and prolapsing mucosa and submucosa, in a proximal-to-distal direction, along a longitudinal axis (Figs. 5, 8). The recommended distance between each suture is approximately $0.5 \mathrm{~cm}$, which is optimal in order to avoid sutures that are too tight (a shorter distance has a lesser plicating effect as well as increased risk of tissue ischemia) or too loose (a longer distance with consequent formation of wide enfolding of rectal mucosa/ submucosa and increased risk of early postoperative rupture of the running suture). While performing MP, when the "marker point" is visualized, the surgeon takes care to make a passage of the running suture above and another below the "marker point," in order to entrap the hemorrhoidal artery within the running suture and accomplish the dearterialization according to the DDD principle (Fig. 9). Each vertical row should be spaced from the adjacent one in order to guarantee enough blood outflow from the hemorrhoids via the venous plexus. In fact, a circumferential obliteration of rectal tissue might create a significant obstacle for the blood and consequently an increased risk of postoperative thrombosis. The MP running suture is stopped at the proximal apex of the internal hemorrhoid, avoiding its inclusion in the mucopexy. When performed this way, the THD method can effectively be considered a hemorrhoid-sparing procedure. Finally, the suture is gently tied (Fig. 10).

\section{Postoperative management}

A diet rich in fluids (oral intake of at least 21 of water per day) and fiber is established, eventually supplemented by oral assumption of stool softeners. Use of laxatives is advisable. In fact, especially in patients who underwent

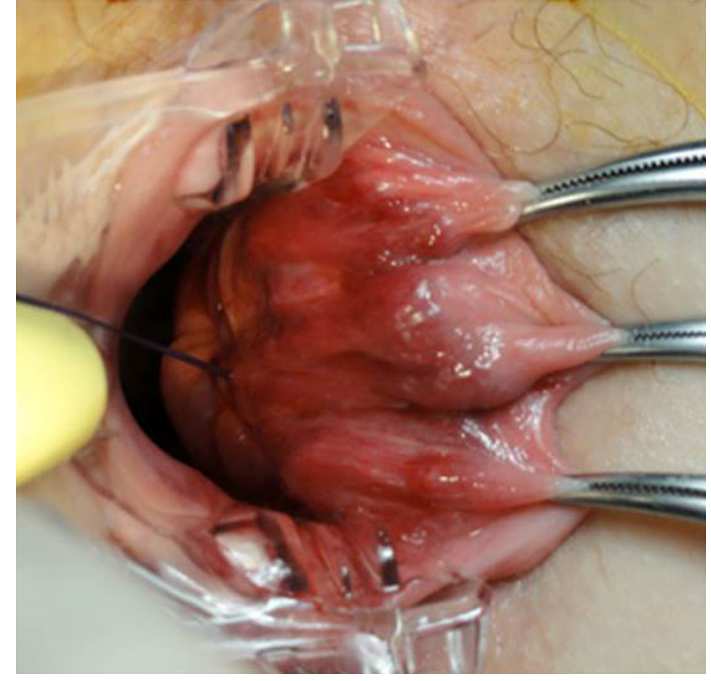

Fig. 10 Mucopexy suture is secured without including the hemorrhoid

MP, not only constipation but also diarrhea and increased frequency of bowel movements could cause an early disruption of the rectal sutures and, then, possible bleeding from the mucopexy suture(s) and early recurrence of prolapse. Scrupulous adherence to a dietary protocol is usually recommended during the first 2-3 postoperative months, and the patient is encouraged to continue a high residue diet after this time period. Patients with either chronic diarrhea or irritable bowel syndrome should be put on a very carefully controlled diet and pre-/probiotics. On the other hand, those with either chronic inflammatory bowel disease or chronic radiation proctitis must continue the specific therapy as prescribed; a sudden worsening of their condition should be diagnosed early and treated. 
Postoperative care should be strongly directed toward the control of pain and tenesmus. The source of these symptoms is the surgical site (not the hemorrhoidal cushions) and is related to the plication of the rectal mucosa/submucosa. This can cause an inflammatory response (with edema and inflammatory reaction) associated with relative ischemia of those tissues, which causes both pain and tenesmus. As a consequence of the inflammatory process, MP patients can have a mucous, sometimes bloody, anal discharge for a few days. When both piles and the sensitive mucosa of the anal pecten are spared during MP (as described above), these are not the source of pain and tenesmus, unless a hemorrhoidal thrombosis has developed. The severity of pain and tenesmus could be dependent not only on the surgical procedure but also on the patient's tolerance level to pain; in that case, their management of these symptoms should be specially tailored. Patients who undergo dearterialization alone usually suffer minor pain and/or rectal discomfort, lasting from a few hours to a few days. In these patients, anti-inflammatory drugs and/or analgesics can be prescribed "as needed." Patients who had MP more frequently report tenesmus and pain. In these patients, non-steroidal anti-inflammatory drugs (NSAIDs) should be given around the clock for at least 3 days, and other analgesics when requested. With these measures, in the author's experience, both edema and related symptoms are reduced. Usually, patients can discontinue this postoperative regimen after a few days, and only a minority of them needs it for more than 7 days.

Urinary retention develops in about $10 \%$ of patients, especially those who undergo MP and males. To prevent this, restriction of excessive intravenous infusion of fluids is advisable. Treatment should consist only in temporary bladder catheterization.

Tenesmus can be accompanied by a transient sensation of urge to defecate. This is usually transient, with resolution within 7-10 days, and does not give rise to any form of persistent urgency, soiling, or fecal incontinence.

\section{Follow-up}

The follow-up includes 4 different time points. At the first visit, 7-10 days after the procedure, a digital anorectal examination is never carried out, but only an external inspection to avoid the risk of pulling on the stitches. At this time, particular attention is also paid to normalizing defecation with diet and laxatives. Usually, bleeding is no longer present. In a minority of patients after MP, some bloody mucus is referred, due to the early postoperative inflammatory process. Inflammation can also determine mild fever along the first 2-3 postoperative days, usually self-limited and responding to anti-inflammatory drugs. Tenesmus can be referred after MP at this time and gradually improves. Only minority of patients still require analgesics. The second follow-up visit is made after 1 month. The patient's anorectum is digitally explored and assessed. Rectal pain, discomfort, and tenesmus should no longer be present. Persistence of these symptoms should be investigated. In case of some hemorrhoidal prolapse is preset or reported, this is suspicious of suture disruption, usually secondary to defecatory dysfunction. Also intermittent, self-limited episodes of bleeding can be indicative to MP disruption. Anal continence should be fully normal. At the 3 month follow-up visit, the patient is also evaluated with anoscopy. At that time, when the procedure is successful, all symptoms are resolved. Volume and appearance of hemorrhoidal cushions are that of patients without hemorrhoidal disease. Persistent or new bleeding or prolapse will require a closer follow-up. Thereafter, patients are contacted by telephone and examined 1 year after surgery. A long-term annual follow-up may be established. If any symptom related to a possible recurrence of hemorrhoidal disease is reported, the patient undergoes digital examination and anoscopy.

\section{Complications and management}

The most common complication is tenesmus, which sometimes can turn into rectal discomfort or pain. It can be managed with analgesics and anti-inflammatory drugs as described above. However, these symptoms rapidly disappear. Rectal bleeding can occur in a very limited number of patients, usually within 2 weeks after the operation. It can be caused by trauma of the rectal mucosa involved in the surgical procedure (especially MP) during prolonged straining, passage of hard stool, or diarrhea. In fact, excessive suture traction can be generated and can lead to breakage. Moreover, the relative tissue ischemia at the level of the MP suture line can result in a limited necrosis of the mucosa/submucosa and consequent bleeding. In both cases, the removal of clots by saline solution lavage (performed with a soft catheter) can usually stop the bleeding. If bleeding continues and increases in frequency and intensity, it is necessary to perform an endoscopic or surgical hemostasis (cauterization, endoclip, and suture).

In the author's experience, THD, performed according to the principles outlined above, is never followed by fecal incontinence and chronic pain. Indeed, anorectal physiology parameters should be unaltered, and anal sphincters should not be injured by this procedure [19].

\section{Recurrences and their management}

In case of recurrence, the treatment decision making is guided by the symptoms. Recurrence of rectal bleeding can 
occur in cases where the dearterialization was not successful in one or more rectal sectors. Severity of bleeding is usually less than in the initial presentation and can be easily managed with medical therapy, rubber band ligation, or new dearterialization under Doppler guidance.

In the majority of cases of recurrent prolapse, the cause seems to be the disruption of MP suture(s) with difficult defecation early in the postoperative period or later due to chronic straining. To prevent this occurrence, an optimal diet and fiber supplements are necessary in case of constipation, or prompt treatment for IBS and IBD symptoms. Patients with recurrent prolapse can be managed conservatively if the prolapse is minimal. Re-do MP is technically possible although other strategies such as excisional hemorrhoidectomy can also be adopted.

\section{Conclusions}

Transanal hemorrhoidal dearterialization is a valid therapeutic option in patients with hemorrhoidal disease. It can provide effective control of symptoms in the vast majority of patients. Accuracy in both dearterialization (using the "DDD" procedure) and mucopexy (repositioning the prolapsing rectal mucosa and submucosa, completely sparing the piles) seems the key to therapeutic success. However, patients must be informed about postoperative management. The limited number and severity of complications makes THD very safe. Finally, THD can be used in case of recurrent disease.

Conflict of interest The author was speaker at a number of congresses/training courses about the THD Doppler procedure.

\section{References}

1. Schuurman JP, Go PM, Bleys RL (2009) Anatomical branches of the superior rectal artery in the distal rectum. Colorectal Dis 11:967-971

2. Aigner F, Bodner G, Gruber H et al (2006) The vascular nature of hemorrhoids. J Gastrointest Surg 10:1044-1050
3. Aigner F, Gruber H, Conrad F et al (2009) Revised morphology and hemodynamics of the anorectal vascular plexus: impact on the course of hemorrhoidal disease. Int $\mathbf{J}$ Colorectal Dis 24:105-113

4. Aigner F, Bonatti H, Peer S et al (2010) Vascular considerations for stapled haemorrhoidopexy. Colorectal Dis 12:452-458

5. Ratto C, Donisi L, Parello A, Litta F, Doglietto GB (2010) Evaluation of transanal hemorrhoidal dearterialization as a minimally invasive therapeutic approach to hemorrhoids. Dis Colon Rectum 53:803-811

6. Giordano P, Overton J, Madeddu F, Zaman S, Gravante G (2009) Transanal hemorrhoidal dearterialization: a systematic review. Dis Colon Rectum 52:1665-1671

7. Pucher PH, Sodergren MH, Lord AC, Darzi A, Ziprin P (2013) Clinical outcome following Doppler-guided haemorrhoidal artery ligation: a systematic review. Colorectal Dis 15:e284-e294

8. Lucha PA (2009) Pathophysiology of hemorrhoidal disease. In: Khubchandani I, Paonessa N, Azimuddin K (eds) Surgical treatment of hemorrhoids. Springer, London, pp 15-17

9. Loder PB, Kamm MA, Nicholls RJ, Philips RK (1994) Haemorrhoids: pathology, pathophysiology and aetiology. Br J Surg 81:946-954

10. Thomson WH (1981) The anatomy and nature of piles. In: Kaufman HD (ed) The haemorrhoid syndrome. Abacus Press, Tunbridge Wells, pp 15-33

11. Shafik A (2009) Surgical anatomy of hemorrhoids. In: Khubchandani I, Paonessa N, Azimuddin K (eds) Surgical treatment of hemorrhoids. Springer, London, pp 7-13

12. Ratto C, Parello A, Donisi L, Litta F, Zaccone G, Doglietto GB (2012) Assessment of haemorrhoidal artery network using colour duplex imaging and clinical implications. Br J Surg 99:112-118

13. Ratto C, Donisi L, Parello A, Litta F, Zaccone G, De Simone V (2012) Distal Doppler-guided dearterialization' is highly effective in treating haemorrhoids by transanal haemorrhoidal dearterialization. Colorectal Dis 14:e786-e789

14. Tagart RE (1974) Haemorrhoids and palpable ano-rectal lesions. Practitioner 212:221-238

15. Haas PA, Fox TA Jr, Haas GP (1984) The pathogenesis of hemorrhoids. Dis Colon Rectum 27:442-450

16. Keighley MR, Williams NS (2008) Haemorrhoidal disease. In: Keighley MR, Williams NS (eds) Surgery of the anus, rectum and colon, 3rd edn. Elsevier, Saunders, pp 321-381

17. Jackson CC, Robertson E (1965) Etiologic aspects of hemorrhoidal disease. Dis Colon Rectum 8:185-189

18. Gass OC, Adams J (1950) Hemorrhoids: etiology and pathology. Am J Surg 29:40-43

19. Ratto C, Parello A, Donisi L, Litta F, Doglietto GB (2011) Anorectal physiology is not changed following transanal haemorrhoidal dearterialization for haemorrhoidal disease: clinical, manometric and endosonographic features. Colorectal Dis 13:e243-e245 